\title{
The innovation of the subspecialty of Paediatric Virology: An interview with Research Professor of Molecular Virology Anna Kramvis
}

\author{
IOANNIS N. MAMMAS and DEMETRIOS A. SPANDIDOS \\ Department of Clinical Virology, School of Medicine, University of Crete, Heraklion 71003, Greece
}

Received July 18, 2017; Accepted August 22, 2017

DOI: $10.3892 /$ etm.2017.5008

\begin{abstract}
Professor Anna Kramvis, Research Professor of Molecular Virology at the University of the Witwatersrand in Johannesburg, South Africa, talks about direct-acting antiviral treatments against hepatitis $\mathrm{C}$ virus (HCV), as well as the perspective of the development of an effective vaccine against HCV. She emphasises the necessity of vaccination against hepatitis B virus (HBV), highlighting that it is very important that vaccination should be administered at birth in order to prevent mother-to-child transmission (MTCT) of HBV. Professor Kramvis states that vaccination against HBV is safe and that HBV and HCV infections are not contraindications for breastfeeding. Regarding the challenge of Paediatric Virology, she believes that it is a field that during the last years is increasing exponentially, while she concurs that Paediatric Virology subspecialty will be a popular choice for infectious diseases subspecialists. In the context of the 3rd Workshop on Paediatric Virology, which will be held in Athens on October 7th, 2017, Professor Kramvis will give her key lecture on MTCT of HBV and HCV.
\end{abstract}

\section{Contents}

1. Introduction

2. Questions and Answers

\section{Introduction}

Professor Anna Kramvis is a Research Professor of Molecular Virology and Director of the Hepatitis Virus Diversity Research Unit, which provides a platform for training of research scientists in Molecular Virology (1). Her primary

Correspondence to: Professor Demetrios A. Spandidos, Department of Clinical Virology, School of Medicine, University of Crete, Heraklion 71003, Greece

E-mail: spandidos@spandidos.gr

Key words: Paediatric Virology, hepatitis B, hepatitis C, Anna Kramvis research interest is the Molecular Virology of the hepatitis viruses, and in particular that of unique African strains of the virus, which differ from those found in other regions of the world (2-5). The focus of her research has been the study of the sequence variation of African hepatitis B virus (HBV) strains, their functional characterisation and their role in the clinical manifestation of disease. In addition to HBV-mono-infection, her team is researching HBV co-infection with human immunodeficiency virus (HIV) in the acquired immunodeficiency symdrome (AIDS) pandemic scourging continent of Africa. She has published over 70 articles in international journals. She has supervised and successfully graduated $\mathrm{PhD}$ and MSc students and has hosted a number of post-doctoral fellows. Her current team consists of 3 postdoctoral fellows, $2 \mathrm{PhD}$ and 7 MSc students. The South Africa's National Research Foundation (NRF) has rated her as an internationally recognised scientist. She is a Fellow of the Academy of Science of South Africa (ASSAf) and an Honorary Research Associate of the Victorian Infectious Diseases Reference Laboratory in Melbourne, Australia. She is a member of the European Association for the Study of the Liver and sits on various international and national committees, including the Hepatitis Transformative Science Group of the AIDS Clinical Trials Group, and the International Committee for the Elimination of HBV (ICE-HBV). Professor Kramvis has established extensive, ongoing, national and international collaborative networks with laboratories in South Africa, Botswana, Kenya, Sudan, Zimbabwe, China, India, Japan, Belgium, Germany, Greece, Sweden, Australia, Brazil and the USA, proof of the importance of hepatitis virus research and its national and international relevance. Together with Professor Jake Liang, she is organiser of the 2017 International HBV Meeting to be held in Washington in September 2017.

To date, Professor Kramvis has chaired the 1st and the 2nd Workshop on Paediatric Virology held in Athens in 2015 and 2016 and has given her key lectures on hepatitis virology in both events $(1,6-8)$. She has been awarded by the Paediatric Virology Study Group (PVSG) with the '2015 Paediatric Virology Award in Virology' and the '2016 Paediatric Virology Award in Hepatitis' for her outstanding academic and clinical research and her exceptional teaching and publishing contribution on hepatitis virology and its study in Africa (1,7). In the context of the 3rd Workshop on Paediatric Virology, which will be held in Athens on October 7th, 2017 (9), Professor Kramvis 
will give her key lecture on mother-to-child transmission (MTCT) of HBV and HCV.

\section{Questions and Answers}

Question: Professor Anna Kramvis, first of all, thank you for your chairship at the 1st and the 2nd Workshop on Paediatric Virology and your continuous support all these years. You are considered as a worldwide expert in hepatitis virology. What are the recent advances in the field of hepatitis virology that a paediatrician should be aware of?

Answer: There are now direct-acting antiviral (DAA) treatments that act against hepatitis $\mathrm{C}$ virus $(\mathrm{HCV})$ that can prevent the replication of HCV and cure HCV infection after 12 weeks of treatment. In April 2017, these drugs were approved by the US Food and Drug Administration for paediatric cases (https://www.fda.gov). The drugs have been approved for use in children 12 years or older or weighing at least $35 \mathrm{~kg}$. The drugs include Sovaldi (sofosbuvir) and Harvoni (ledipavir and sofosbuvir), which are marketed by Gilead Sciences, Inc. (Foster City, CA, USA). The most common adverse events observed following treatment included fatigue and headaches. Sovaldi was evaluated in children in combination with ribavirin. Paediatricians must check for HBV co-infection, as DAA treatment can lead to HBV reactivation, which can result in serious liver problems or even death. A modification on an agreed Paediatric Investigation Plan (PIP) has been submitted to the European Medicines Agency (http://www.ema.europa.eu).

Question: Should vaccination against HBV be given to newborns instead of two-month-old babies?

Answer: The earlier a child is infected with HBV, the greater the risk of chronic infection, which can manifest in cirrhosis and/or liver cancer (hepatocellular carcinoma) in adulthood. The frequency of chronic infection is $90 \%$ if newborns are infected, whereas it is only $5 \%$ if infected in adulthood. Children can be infected antenatally, perinatally or horizontally. MTCT can occur by all three modes, but most frequently, perinatally. In total, 70 to $90 \%$ of infants born to HBeAg-positive mothers will become chronic HBV carriers within 3 months of birth. Therefore, it is very important that vaccination is administered as soon as possible to prevent both MTCT and the development of chronic carriage. The World Health Organisation (WHO) has recommended that all neonates receive the first dose of the hepatitis B vaccine within $24 \mathrm{~h}$ of birth. Ideally, it should be administered in the delivery room. If the mother is HBsAg-positive the vaccine must be given together with the administration of hepatitis B immune globulin (HBIg). HBIg can be given from 28 weeks gestation to the mother or to the newborn at birth. The vaccine plus HBIg will not, however, prevent infection that occurred in utero.

Question: What would your advice be to a parent who has concerns about the adverse reactions of the vaccine against HBV?

Answer: The hepatitis B vaccine is safe and like any other vaccine may relatively rarely have some side-effects. Considering the current evidence, the risks of HBV infection, and its consequences, far exceed the theoretical risks that could be induced by the vaccine.

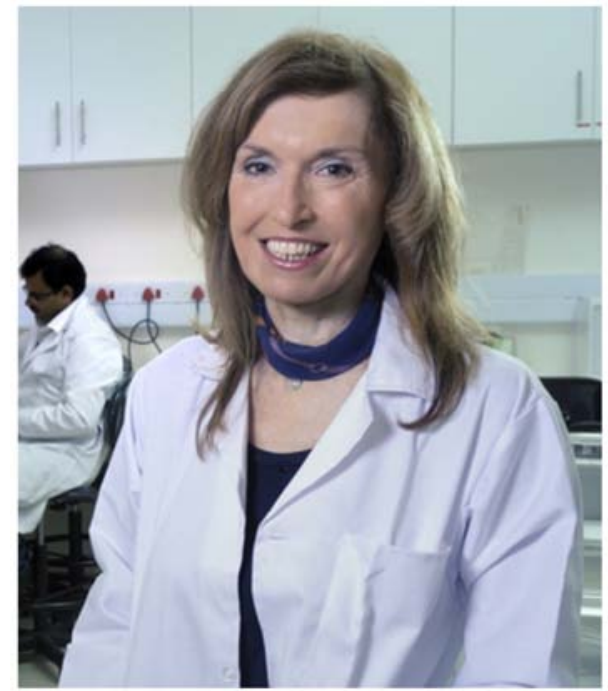

Figure 1. Professor Anna Kramvis, Research Professor of Molecular Virology at the University of the Witwatersrand in Johannesburg, South Africa.

Question: Are hepatitis viruses transmitted via human breast milk? Is hepatitis virus infection a contraindication for human breastfeeding?

Answer: $\mathrm{HBsAg}$ has been detected in $71 \%$ of breast milk samples and MTCT can occur during childcare activities, including breastfeeding, although studies have shown no difference between transmission from HBsAg-positive mothers to infants that were breast-fed compared to those that were bottle-fed. Thus, neither HBV nor HCV infection are contraindications for breastfeeding.

Question: A future vaccine against $\mathrm{HCV}$ would radically limit hepatitis $\mathrm{C}$ in humans. What are the chances for the development of such a vaccine?

Answer: The availability of DAAs that can cure the majority of treated HCV-infected patients has been a great success story in hepatitis research and development. However, the eradication of $\mathrm{HCV}$, as for any other infectious disease, requires effective preventative measures including the development of a vaccine. $\mathrm{HCV}$ is a major blood borne viral infection, for which there is no vaccine. $\mathrm{HCV}$ is a positive-sense single-stranded RNA virus belonging to the family Flaviviridae, which is characterised by sequence heterogeneity. This heterogeneity, together with the fact that there is no immunocompetent small animal model that can be used to study this virus, have hampered efforts for vaccine development. A number of prophylactic $\mathrm{HCV}$ vaccines have been shown to be safe and well tolerated in phase I human trials and the completion of phase II trials is expected by early 2018. Although there is some reserved optimism, the development of an effective vaccine is a goal that will be relatively difficult to achieve.

Question: In your last lecture at the 2nd Workshop on Paediatric Virology, you noted that if we desire to eradicate hepatitis globally by 2030 that is in 15 years, hepatitis research has to be given the attention it deserves. What are the challenges that you face in your research unit in order to achieve that goal?

Answer: My research unit is based in South Africa, where hepatitis research has been eclipsed by what are known as the 
'blockbuster' infections that cause AIDS, malaria and tuberculosis. This has meant that additional funding was not available for hepatitis research, which was considered to be preventable, at least in the case of HBV. However, our research and that of others, has shown that HBV infection is not a minor issue, particularly in HIV-infected individuals, where HBV/HIV co-infection is frequent and is also characterised by an infection that cannot be detected by conventional serological assays, as the infection is HBsAg-negative. Fortunately, the tide is turning for previously neglected and under-funded fields of research in hepatitis. Two recent major developments have highlighted the importance of hepatitis research and put it in the spotlight: i) In May 2016, the adoption by the WHO of the first global health sector strategy (GHSS) on viral hepatitis 'Towards ending viral hepatitis, 2016-2021' in order to eliminate viral hepatitis as a major public health threat by 2030 and ii) in 2013, the revolutionary development of the first DAAs for HCV treatment. Thus, funding agencies are becoming more enthusiastic and keen to support our research, particularly in Africa, from where unprecedented human migrations have made hepatitis a global rather than an African problem.

Question: 'As more than $90 \%$ of the cases attended to in a paediatric unit are children with a viral infection, knowledge of Virology is important in Paediatrics'. Please share your view on this statement.

Answer: Viral infections differ from bacterial infections in terms of the microorganisms causing the disease, the natural history of the infection and in the way that the infections can be prevented and treated. A paediatrician needs to be aware of these differences. Moreover, viruses can have unique characteristics, which the paediatrician may need to be aware of, in order to be able to manage the infections. To illustrate this, if we compare HBV and HCV, although the viruses both cause hepatitis, they are completely different viruses. $\mathrm{HBV}$ is a partially double stranded DNA virus, whereas $\mathrm{HCV}$ is a single stranded positive strand RNA virus. They differ in both their replication and natural history. There is an effective vaccine against HBV, so this infection can be prevented, whereas there is no vaccine against HCV. On the other hand, we have effective DAAs that can cure HCV infection. Although there are effective antiviral treatments that can reduce HBV viral loads, there is currently no antiviral treatment that can completely eliminate HBV.

Question: How would you evaluate our proposal of Peadiatric Virology as a new paediatric subspecialty candidate? Could this proposal add new issues in medical education of undergraduates medical students as well as paediatric trainees?

Answer: Paediatric Virology is a field that during the last years is increasing exponentially. The advances in Molecular Virology, the new treatments that are available, as well as the unprecedented human migrations, we are experiencing at present, mean that the field is growing in a number of directions: Molecular Biology, Pharmaceutics and Epidemiology.
Added to this we also have the emergence of new or previously unrecognised viral infections, as for example, Zika virus, H5N6 influenza virus, Ebola virus, new HIV outbreaks, just to mention a few. I think that the recognition of Paediatric Virology as a subspeciality will allow this area that is often neglected to grow and research in this field to be supported. The paediatric population is probably our most vulnerable age group and our future. Therefore, it is important that their health is prioritised. Paediatric Virology, as a subspecialty, will allow for an understanding of this field as well as research in areas, which would benefit this age group. Often the drugs that are available for adults cannot be used in the paediatric population and these have to be customised for the paediatric population. Although vaccines are available for many childhood viral infections, often the political will is not available to allow optimal implementation and coverage of the vaccination in many resource limited environments. I believe that Paediatric Virology subspecialty will be a popular choice for infectious diseases subspecialists. A healthy balance should be maintained between overspecialising and being knowledgeable of the field in general.

Question: Thank you for your detailed and encouraging answers; we are looking forward to your key lecture on MTCT of HBV and HCV at the 3rd Workshop on Paediatric Virology on October in Athens.

\section{References}

1. Greenough A, Theodoridou M, Kramvis A, Mammas IN, Christaki I, Koutsaftiki C, Koutsaki M, Kostagianni G, Portaliou D, Papadopoulou P, et al: Workshop on Paediatric Virology. Paediatric Virology Study Group, Athens, October 10, 2015.

2. Kramvis A: The clinical implications of hepatitis B virus genotypes and $\mathrm{HBeAg}$ in pediatrics. Rev Med Virol 26: 285-303, 2016.

3. Kramvis A: Genotypes and genetic variability of hepatitis B virus. Intervirology 57: 141-150, 2014.

4. Kramvis A and Clements CJ: Implementing a birth dose of hepatitis B vaccine for home deliveries in Africa - too soon? Vaccine 28: 6408-6410, 2010.

5. Kramvis A, Kew M and François G: Hepatitis B virus genotypes. Vaccine 23: 2409-2423, 2005.

6. Mammas IN, Greenough A, Theodoridou M, Kramvis A, Christaki I, Koutsaftiki C, Koutsaki M, Portaliou DM, Kostagianni G, Panagopoulou P, et al: Current views and advances on Paediatric Virology: An update for paediatric trainees. Exp Ther Med 11: 6-14, 2016.

7. Thiagarajan P, Gardner S, Theodoridou M, Kramvis A, Melidou A, Papaioannou G, et al: 2nd Workshop on Paediatric Virology. Paediatric Virology Study Group, Athens, October 8, 2016.

8. Mammas IN, Theodoridou M, Kramvis A, Thiagarajan P, Gardner S, Papaioannou G, Melidou A, Koutsaki M, KostagianniG, Achtsidis V, et al: Paediatric Virology: A rapidly increasing educational challenge. Exp Ther Med 13: 364-377, 2017.

9. Mammas IN and Spandidos DA: Athens-based meeting to discuss the paediatric virology crossroad in October 2017. Acta Paediatr 106: 1536, 2017. 